\title{
Complete eradication of de-differentiated skin recurrence of papillary thyroid carcinoma with weekly docetaxel
}

\author{
Naoyoshi Onoda $\cdot$ Hidemi Kawajiri $\cdot$ Shinichiro Kashiwagi $\cdot$ Masanori Nakamura \\ Tsutomu Takashima $\cdot$ Masahiko Osawa $\cdot$ Kenichi Wakasa $\cdot$ Tetsuro Ishikawa • \\ Kosei Hirakawa
}

Received: 10 September 2012/Accepted: 29 October 2012/Published online: 5 December 2012

(C) The Japan Society of Clinical Oncology 2012

\begin{abstract}
Standardized therapeutic strategies have not been established yet to manage poorly to undifferentiated thyroid cancer. We experienced a 74-year-old female patient with advanced papillary thyroid cancer that recurred in the cervical skin with a de-differentiation profile after the initial extended surgical therapy. The patient was treated with weekly administration of docetaxel. A rapid regression of the lesion was observed clinically, and complete eradication of the lesion was confirmed pathologically after 14 courses of the treatment. The residual well-differentiated papillary thyroid carcinoma compartment was pathologically confirmed in two cervical lymph nodes after chemotherapy. Although no further treatment was conducted, the patient had no sign of recurrent disease until an accident caused her death 45 weeks after the initial operation. Systemic chemotherapy with weekly administration of docetaxel might be one of the strategies worth implementing to manage patients with de- or poorly differentiated papillary thyroid carcinoma.
\end{abstract}

Keywords Thyroid cancer - De-differentiation . Chemotherapy · Docetaxel

N. Onoda $(\bowtie) \cdot$ H. Kawajiri · S. Kashiwagi · M. Nakamura .

T. Takashima $\cdot$ T. Ishikawa $\cdot$ K. Hirakawa

Department of Surgical Oncology, Osaka City University

Graduate School of Medicine, 1-4-3 Asahi-machi,

Abeno-ku, Osaka 545-8585, Japan

e-mail: nonoda@med.osaka-cu.ac.jp

M. Osawa $\cdot$ K. Wakasa

Department of Diagnostic Pathology, Osaka City University Graduate School of Medicine, 1-4-3 Asahi-machi, Abeno-ku, Osaka 545-8585, Japan

\section{Introduction}

No standardized therapeutic strategy has been established for the patients with recurrent thyroid carcinoma, especially when the lesion shows a de-differentiation profile from the initial differentiated thyroid carcinoma [1]. Practically, individual management is adopted as a challenging therapy. Although various methods of treatment have previously been attempted, the efficacies of these managements have hardly been demonstrated. We experienced a case of thyroid carcinoma showing de-differentiating recurrence in the cervical skin after the initial surgical management for an advanced papillary thyroid carcinoma (PTC). Using scheduled chemotherapy according to the weekly docetaxel regimen, the de-differentiated compartment of the lesion completely disappeared, leaving a limited metastatic PTC compartment within two cervical lymph nodes. The pathologically confirmed complete response lasted until the patient died from an accident. In this report, we describe the case and discuss prospects for the appropriate management of patients with de-differentiated thyroid carcinoma.

\section{Case report}

A 74-year-old female was referred to our institute for the further management of advanced thyroid carcinoma. A computed tomographic examination revealed a huge mass extending from the left lobe of the thyroid to the upper mediastinum severely compressing the trachea (Fig. 1). Several lymph node swellings suggesting metastases were found in the mediastinum. No distant metastasis was demonstrated by imaging studies. Blood counts and thyroid function values were within the normal range. The serum 


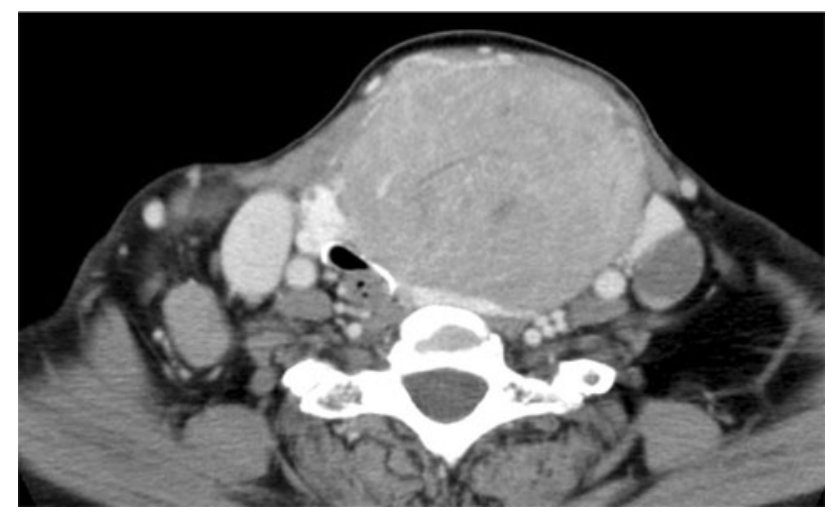

Fig. 1 A computed tomographic examination revealed a huge mass extending from the left lobe of the thyroid to the upper mediastinum severely compressing the trachea and large vessels in the mediastinum

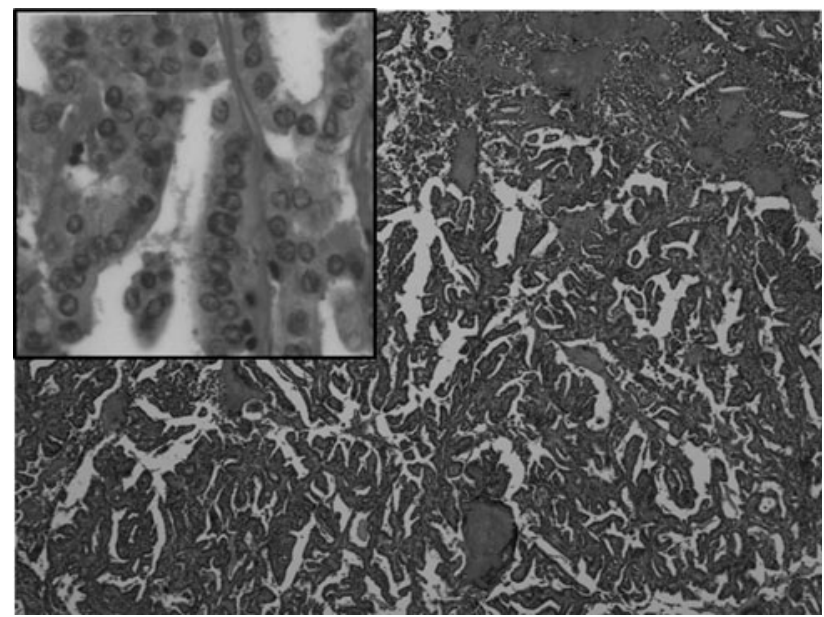

Fig. 2 The initially resected tumor consisted mainly of differentiated PTC

thyroglobulin level was elevated to $4,340 \mathrm{ng} / \mathrm{ml}$. The cytological examination revealed PTC. Therefore, an extended surgical resection was conducted. A total thyroidectomy operation with lymph node dissection was successfully undertaken; it was associated with partial resection of the superior vena cava and the esophageal wall, and shaving of the left recurrent laryngeal nerve. The tumor consisted of PTC (Fig. 2). Histologically, no undifferentiated carcinoma compartment was recognized.

Radioisotope ablation therapy (RIA) was postponed because of the second surgery for axillar lymph node recurrence revealing metastatic PTC in the lymph nodes. RIA was again postponed because of skin metastasis spread over the neck occurring 24 months after the initial operation (Fig. 3a). The biopsy demonstrated poorly differentiated carcinoma invading subcutaneously (Fig. 3b). Immunostaining showed that the cancer cells were weakly positive for thyroglobulin. Multiple cervical lymphadenopathies suggestive of metastases were shown by ultrasonography. The patient required systemic therapy because of the disseminating poorly differentiated carcinoma in the neck. A chemotherapeutic treatment with once a week administration of $25 \mathrm{mg} / \mathrm{m}^{2}$ of docetaxel (DOC) was initiated after obtaining written informed consent. A rapid regression of redness and swelling of the skin lesion was noticed within two courses. However, a grade 2 peripheral neuropathy appeared on the 13th course and gradually progressed. The skin lesion disappeared after the 14th course had been completed (Fig. 3c), and no cancer cells were demonstrated by the repeat biopsy (Fig. 3d). Moreover, the lymphadenopathies disappeared, leaving two nodes in the right lateral neck. The chemotherapy was terminated because of an adverse effect of grade 3 peripheral neuropathy. The lateral neck lymph nodes gradually enlarged. No other cancerous lesions were demonstrated by the positron emission computed tomography. Therefore, these two nodes were excised, and the metastatic cancer nest consisting only of well-differentiated PTC was confirmed pathologically (Fig. 4).

The patient was well, and no recurrent disease had been identified with TSH suppression at 45 months after the initial operation, when she died because of an accident of causes unrelated to the disease itself or the adverse effects of chemotherapy.

\section{Discussion}

Recurrent disease can be found in approximately $30 \%$ of the patients with PTC [2]. Approximately $5 \%$ of these patients demonstrated de-differentiation profiles in the recurrent focus [1] and displayed poor outcomes after showing resistance to conventional therapeutic strategies [3]. In these cases further therapy with TSH suppression or RIA is not more effective; thus, various methods and anticancer drugs have been studied in attempts to manage the disease $[4,5]$. Still, no standard therapeutic strategy could be established to manage de-differentiated, poorly or undifferentiated thyroid cancer because of the lack of objective or reliable evidence.

Several reports have demonstrated a clinical benefit of DOC-based chemotherapy for patients with iodine-refractory de-differentiated PTC $[6,7]$. DOC has been widely used in patients with breast, gastric, and head and neck cancer, and the efficacy of the drug was confirmed to be promising. Therefore, we have chosen DOC to manage poorly to undifferentiated PTC at our institute since 2000. We have attempted to use DOC in combination with extrabeam radiation therapy and found some effect on the local control to avoid suffocation or bleeding. In the present case, we found a significant effect of DOC on 

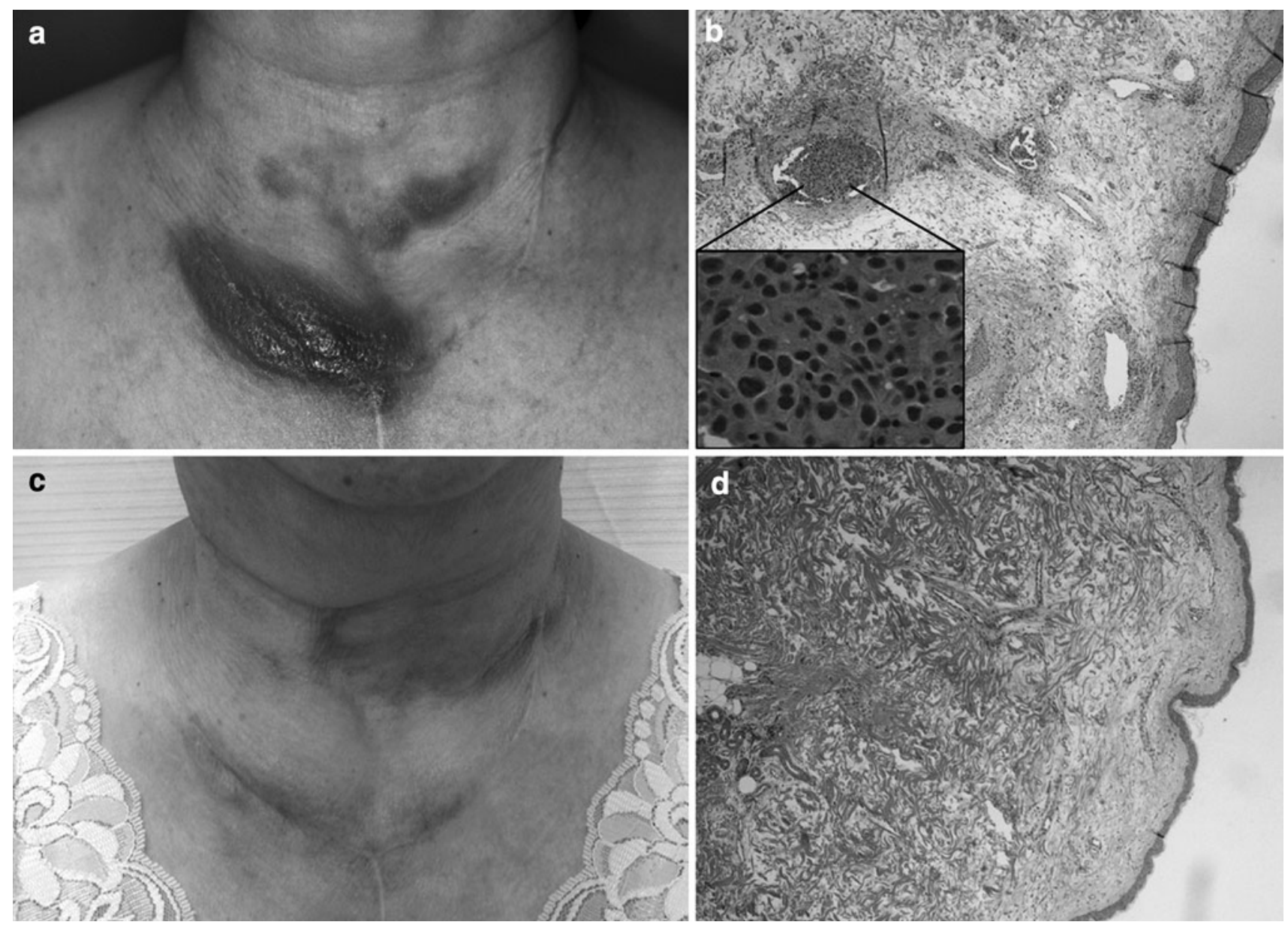

Fig. 3 Skin metastasis occurring 24 months after the initial operation (a). Biopsy of the skin demonstrated poorly differentiated carcinoma (b). The skin lesion disappeared after the 14th course of weekly DOC

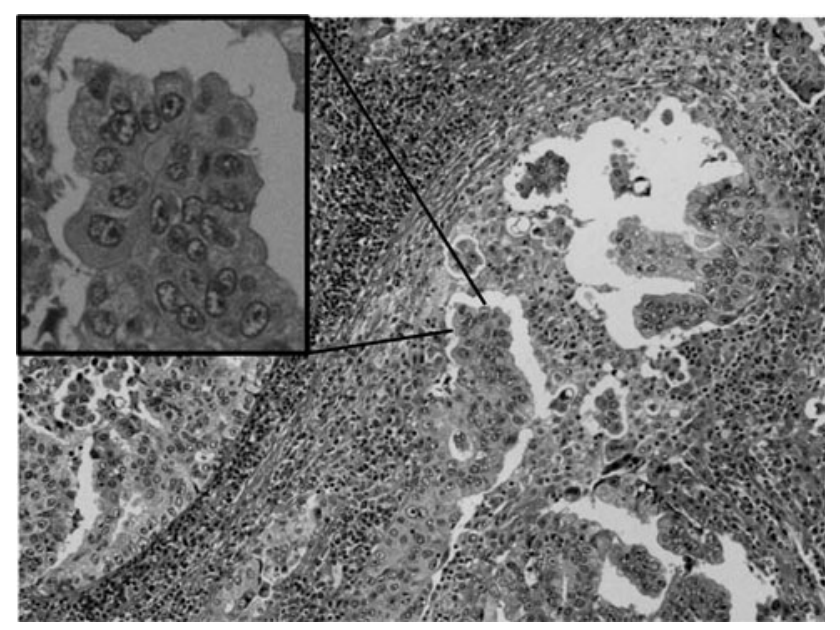

Fig. 4 The metastatic cancer nest, left in cervical lymph nodes after chemotherapy, consisted only of the PTC component

de-differentiated recurrence in the skin and the majority of the cervical lymph nodes. Our case is the first to confirm the pathological evidence of complete eradication of had been completed (c), and no cancer cells were demonstrated by the repeated skin biopsy (d)

de-differentiated PTC. Interestingly, lesions in two remaining lymph nodes consisted only of PTC, indicating resistance to chemotherapy in these foci. Our observation clearly demonstrated that the difference of sensitivity to chemotherapy may be determined, in part, by the grade of differentiation in PTC [8].

One of the main adverse effects of the DOC was neuropathy [9]. Approximately 5-50\% of the patients suffered from peripheral neuropathy, and this was associated with the cumulative dose of the anti-cancer agent. No remarkable effect to overcome the toxicity has been found. Thus, in some cases, as in this one, neuropathy might become a dose-limiting factor [9].

Several strategies have been attempted to manage de-differentiated thyroid cancer, such as introducing re-differentiation or targeting altered molecules. Experimental studies could demonstrate the potential of these strategies as therapeutic methods [10]. However, no remarkable clinical effect could be obtained with re-differentiation therapy [11]. Several tyrosine kinase inhibitors have been applied to manage de-differentiated thyroid 
cancer, such as sorafenib, vandetanib, or pazopanib, to inhibit molecular pathways contributing to cancer growth, demonstrating significant but limited effects in clinical trials $[5,12]$. A certain period is still required for the practical clinical application of newly developed molecular targeted therapy. Our case suggests the possible application of DOC-based chemotherapy to manage de-differentiating and poorly differentiated carcinoma of the thyroid as one of the possible practical therapies.

Acknowledgment This report was supported, in part, by JSPS Grant-in-Aid for Scientific Research (C) (2010-12) \#22591439.

Conflict of interest The authors declare that they have no conflict of interest.

\section{References}

1. Antonelli A, Fallahi P, Ferrari SM et al (2008) Dedifferentiated thyroid cancer: a therapeutic challenge. Biomed Pharmacother 62:559-563

2. Cooper DS, Doherty GM, Haugen BR, American Thyroid Association (ATA) Guidelines Taskforce on Thyroid Nodules and Differentiated Thyroid Cancer et al (2009) Revised American Thyroid Association management guidelines for patients with thyroid nodules and differentiated thyroid cancer. Thyroid 19: $1167-1214$
3. Baudin E, Schlumberger M (2007) New therapeutic approaches for metastatic thyroid carcinoma. Lancet Oncol 8:148-156

4. Cabanillas ME, Waguespack SG, Bronstein Y (2010) Treatment with tyrosine kinase inhibitors for patients with differentiated thyroid cancer: the M. D. Anderson experience. J Clin Endocrinol Metab 95:2588-2595

5. Benvenga S (2011) Emerging therapies in sight for the fight against dedifferentiated thyroid cancer. J Clin Endocrinol Metab 96:347-350

6. Ikeda M, Tanaka K, Sonoo H et al (2007) Docetaxel administration for radioiodine-resistant patients with metastatic papillary thyroid carcinoma. Gan To Kagaku Ryoho 34:933-936 (in Japanese)

7. Tulloch-Reid M, Skarulis MC, Sherman SI et al (2009) Longterm eradication of locally recurrent invasive follicular thyroid carcinoma after taxane-based concomitant chemoradiotherapy. Anticancer Res 29:4665-4671

8. Burns WR, Zeiger MA (2010) Differentiated thyroid cancer. Semin Oncol 37:557-566

9. Argyriou AA, Koltzenburg M, Polychronopoulos P et al (2008) Peripheral nerve damage associated with administration of taxanes in patients with cancer. Crit Rev Oncol Hematol 66:218-228

10. Smallridge RC, Marlow LA, Copland JA (2009) Anaplastic thyroid cancer: molecular pathogenesis and emerging therapies. Endocr Relat Cancer 16:17-44

11. Kebebew E, Peng M, Reiff E et al (2006) A phase II trial of rosiglitazone in patients with thyroglobulin-positive and radioiodine-negative differentiated thyroid cancer. Surgery 140:960966 (discussion 966-967)

12. Antonelli A, Ferri C, Ferrari SM et al (2010) New targeted molecular therapies for dedifferentiated thyroid cancer. J Oncol 2010:921682 